\title{
Stability investigation of an airfoil section with active flap control
}

\author{
Bergami, Leonardo; Gaunaa, Mac
}

Published in:
Wind Energy

Link to article, DOI:

10.1002/we.354

Publication date:

2010

Link back to DTU Orbit

Citation (APA):

Bergami, L., \& Gaunaa, M. (2010). Stability investigation of an airfoil section with active flap control. Wind Energy, 13(2-3), 151-166. https://doi.org/10.1002/we.354

\section{General rights}

Copyright and moral rights for the publications made accessible in the public portal are retained by the authors and/or other copyright owners and it is a condition of accessing publications that users recognise and abide by the legal requirements associated with these rights.

- Users may download and print one copy of any publication from the public portal for the purpose of private study or research.

- You may not further distribute the material or use it for any profit-making activity or commercial gain

- You may freely distribute the URL identifying the publication in the public portal

If you believe that this document breaches copyright please contact us providing details, and we will remove access to the work immediately and investigate your claim. 


\title{
Stability investigation of an airfoil section with active flap control
}

\author{
Leonardo Bergami, Mac Gaunaa \\ Technical University of Denmark, Wind Energy Department, Roskilde, 4000 Denmark \\ leob@dtu.dk \\ This is the accepted version of the following article: Bergami, Leonardo and Mac Gaunaa. \\ 'Stability investigation of an airfoil section with active flap control.' Wind Energy 13, no. \\ 2-3 (2010):151-166. doi:10.1002/we.354, which has been published in final form at Wiley \\ Online Library
}

\begin{abstract}
This paper presents a method to determine flutter and divergence instability limits for a $2 \mathrm{D}$ airfoil section fitted with active control. The control system consists of a flap-like deformable trailing edge actuator, maneuvered by algorithms based on measurements of either heave displacement, local angle of attack, or pressure difference over the airfoil. The purpose of the trailing edge actuator is to reduce fluctuations in the aerodynamic forcing and a significant potential for active fatigue load alleviation has been reported in recent studies.

Besides the control, the full model of the flap equipped airfoil also comprises a structural and an aerodynamic part. The in-plane motion and deformation of the $2 \mathrm{D}$ structure are described by three degrees of freedom: heave translation, pitch rotation and flap deflection. A potential flow model provides the aerodynamic forces and their distribution, the unsteady aerodynamics are described using an indicial function approximation. Stability of the full aeroservoelastic system is determined through eigenvalue analysis.

Validation is carried out against a reimplementation of the recursive method by Theodorsen and Garrick for 'flexure-torsion-aileron' flutter. The implemented stability tool is then applied to an airfoil section representative of a wind turbine blade with active flap control. It is thereby observed that the airfoil stability limits are significantly modified by the presence of the flap, and they depend on several parameters: flap structural characteristics, type of control, control gain factors and time lag.
\end{abstract}


Keywords: Aeroservoelastic Instability, Flutter, Deformable Flap Control.

\section{Introduction}

An important contribution to the fatigue loads that a blade experiences on a modern horizontal axis wind turbine (HAWT) originates from fluctuations in the aerodynamic forcing, as produced, for instance, by wind turbulence, wind shear, tower shadow or yaw misalignment. The possibility of reducing such contribution by means of active control systems has been addressed in several research projects.

Bossanyi [1] and Larsen et al. [2] investigate the fatigue load alleviation potential of an improved blade pitch control. Larsen et al. [2] reach to the conclusion that by adopting a cyclic blade pitching the flapwise fatigue loads can be reduced up to $15 \%$ with respect to the normal collective pitch, and a 28 $\%$ reduction would be instead achieved by pitching each blade independently. Pitching the whole blade, though, may become inadequate as HAWT blades increase in size and become more flexible. Further load reduction would hence require a faster control, able to intervene locally along the blade span.

As concluded in the overview given by Barlas and van Kuik [3], a significant reduction of the fatigue loads could be achieved through a 'smart' rotor, fitted with active aerodynamic control systems. At the state of the art, several studies report considerable potentials for aerodynamic control methods based either on deploying tabs [4], or on classic rotating flaps [5] or on flap-like deformable trailing edges $[6,7,8,9,10,11]$. Unlike a classic rigid plain flap, a deformable trailing edge flap deflects the aft part of the airfoil following a non-linear deformation shape, with a smooth and continuous variation of the camberline slope, thus avoiding discontinuity points as the hinge in a classic rotating flap. CFD studies by Troldborg [7] have shown that this is beneficial in terms of the efficiency of the airfoil, measured as the lift-to-drag ratio, compared to the classic hinged flap case. Furthermore, it is believed to be beneficial in terms of noise production as well. Buhl et al. [6] simulate the response of a 2D airfoil where a deformable trailing edge flap is controlled in order to compensate for variations in the incoming turbulent flow, thus achieving a considerable reduction in the lift fluctuations. The aerodynamic forces in the simulations by Buhl et al. [6] are computed employing Gaunaa's [12] potential flow model for a thin airfoil section undergoing arbitrary motion and camberline deformation. Andersen et al. [8] extends the model to take into account the effects from fluid viscosity and simulations including the interaction with a wind turbine standard control system are presented in Andersen et al. [9]. Here, it is concluded that by apply- 
ing an active flap control the blade flapwise fatigue load would be reduced up to $48 \%$, with respect to an HAWT without flap-like deformable trailing edges.

Active control with deformable trailing edge flap actuators has shown a significant potential for fatigue load alleviation. Nevertheless, the application of such device on a wind turbine blade also modifies the aeroelastic stability proprieties of the blade itself. A first obvious consequence is a variation in the mass distribution along the airfoil section. A heavy trailing edge flap moves aft the center of gravity of the whole section, thus resulting in lower stability limits, as known from 'classic' stability investigations of rigid wing sections [13, 14, 15]. Furthermore, as observed by Gaunaa [16] for a rigid controlled trailing edge flap, the presence of the flap control also induces variations of the airfoil stability limits that are less trivial and eventually result in significant reduction of the flow speeds at which instabilities are expected to occur. A widened investigation on the stability aspects related to flap control is here presented, focusing the analysis on those aeroelastic instabilities that occur under attached flow conditions: flutter and divergence.

Divergence is a static instability that occurs when the aerodynamic pitching moment, which acts on the airfoil section with a magnitude proportional to the flow speed squared, exceeds the restoring torsion elastic moment, thus leading to extreme airfoil torsions bounded only by nonlinear effects such as stall or nonlinear geometric stiffness. Divergence only depends on the equilibrium between static pitching moments. Therefore, the divergence limit for rigid airfoil sections can be computed with a simple analytical equation, as shown by Theodorsen [13]. A flap equipped section may also undergo another static aeroelastic phenomenon, referred to as control reversal. In fact, the effect of the flap control on the lift force variation is reversed above a certain flow speed, as a consequence of the angle of attack variation caused by the aerodynamic pitching moment produced by the flap deflection, Bisplinghoff et al. [15]. Unlike divergence, control reversal does not necessarily lead to an unstable condition.

Flutter is a more complex dynamic phenomenon and involves the coupling of two or more degrees of freedom, typically heave and torsion. A structure undergoing flutter instability presents self-sustained oscillations around an equilibrium state, the amplitude of the oscillations increases exponentially in time up to a limit cycle, constrained to finite amplitudes only by the system nonlinearities. The flow speed limit at which flutter occurs is usually lower than the divergence one and can not be determined by simple analytical equations, and, although empirical expressions have been proposed [14], generally more complex numerical methods are required.

Theodorsen [13], Theodorsen and Garrick [14] propose a recursive method to determine the flutter limits of a 'typical section' equipped with a conventional 
trailing edge flap. The section is described as a flat plate with infinite span and three degrees of freedom (DOF), the first two being the linearly elastically constrained heave and torsion modes of the main wing. The third DOF is the deflection angle of the flap, which consists of a rigid plate that rotates around its hinge point, also constrained by a linear spring. In Theodorsen and Garrick's formulation, the effects on the aerodynamic forces from the vorticity shed into the wake are modeled by complex Bessel functions that depend on the flutter frequency itself, therefore a recursive solution is required to determine the stability limit. A similar formulation is adopted by Lobitz [17], who modifies the model to determine the flutter limits of an isolated wind turbine blade rotating in still air. Lobitz concludes that, for a 1.5 MW wind turbine blade, flutter would occur if the rotor over-speeds up to double the nominal rotational speed. A similar figure is reported in Hansen's [18] stability investigations for the 5 MW NREL reference turbine, and he also concludes that, concerning flutter instability, the analysis of an isolated blade returns the same results as a full turbine investigation. In Hansen's [19] analysis the effects of the shed wake are modeled using an indicial function approximation, the aeroelastic system is then represented in a state-space formulation and stability is determined using an eigenvalue approach.

The present paper proposes a method to determine the flutter and divergence limits of a 2D airfoil section equipped with deformable trailing edge flap control. The model of the airfoil aeroservoelastic system comprises three interacting parts: structural, aerodynamic and control. The aerodynamic model consists of Gaunaa's [12] thin airfoil unsteady potential flow model, where the effects of the shed wake are represented through an indicial function approximation and the aerodynamic variables are thereby expressed in a state-space formulation. Comparisons with CFD simulations [7] have shown that the aerodynamic model, despite the simplifying assumptions, is able to capture the underlying physics. The control part of the model is then given by a control algorithm and a control delay model, the latter implemented through a first order filter. Three control algorithms are here presented, based on measurements of heave displacement, angle of attack (as in Buhl et al. [6]), or pressure difference between the suction and pressure sides of the airfoil. The airfoil structure is described by a three degrees of freedom model: one DOF for the heaving (plunging) translation, one for the pitch (torsion) rotation and one for the flap deflection, where a generalized coordinate approach is applied to cope with the non-linear deflection shape.

The motion of the airfoil in the streamwise (horizontal) direction is neglected on the ground of results from a preliminary study, briefly presented in the following section. Stability analysis of a rigid airfoil without flap have in fact shown 
that, in the investigated cases, the streamwise degree of freedom scarcely affects the flutter stability limits, and can therefore be neglected, thus simplifying the aerodynamic model to a linear formulation.

Stability of the full aeroservoelastic system in its time-space formulation is then investigated using an eigenvalue approach, a stability tool is thereby implemented to compute flutter and divergence limits of a flap controlled elastically mounted airfoil section. The tool is validated by comparison with a reimplementation of Theodorsen's [13] recursive method for the 'flexure-torsion-aileron' flutter problem and against time marching solutions.

In the last part of the paper, the implemented stability tool is applied to an airfoil, representative of an HAWT blade section, where a deformable trailing edge flap control is introduced for fatigue load reduction. The investigated airfoil section is the same considered in the load alleviation simulations from Buhl et al. [6]. It is thereby possible to assess the effects of a deformable flap on the system stability and whether such control system might critically modify the stability limits.

\section{Preliminary study: undeformable airfoil}

A stability investigation has been first carried out on a simpler aeroelastic system: a 2D airfoil section performing only in-plane rigid body motion. The model, simplified by the absence of the flap, allows to better assess the influence of different parameters on the section stability limits. The results justify the assumption of negligible effects of the streamwise DOF on flutter velocities, as assumed in the flap controlled model, and they generally provide a convenient background and term of comparison for the following investigation of the flap controlled section. The analysis is only briefly outlined here, a more detailed description is reported in Bergami [20].

\subsection{Model and Method}

The rigid in plane motion of the airfoil section is completely described by three degrees of freedom (DOF): two for translations, in the heave and streamwise directions, and one for the rigid body rotation corresponding to the torsional DOF for a full 3D wing. The rotation is defined with respect to the elastic axis, which intersects the airfoil section at the rotation hinge point. Each degree of freedom is then constrained by a linear elastic spring and a viscous damper. The aerodynamic model is based on the state-space formulation presented by Hansen et al. [21], but only the case of fully attached flow is here considered. Due to the streamwise DOF, non linear equations are required to describe the 
aerodynamic forces, and therefore the aeroelastic system needs to be linearized with respect to a specified equilibrium state prior to an eigenvalue stability analysis. Linearized expression for the aerodynamic forces are reported for the general case by Hansen et al. [21] and are tailored to the specific one in Bergami [20]. Stability limits are then computed for a range of heave-torsion frequency ratios and a time marching solution is carried out on the full non linear system to verify the stability limits obtained through the eigenvalue analysis of the corresponding linearized system.

\subsection{Validation}

The model and its implementation are validated by computing the flutter limits for a flat plate, the results are then compared against the figures obtained with a reimplementation of the recursive method for the 'flexure-torsion' flutter problem described by Theodorsen and Garrick [14, 13]. The results computed with the actual stability tool (Figure 1, full thick lines) and the reimplemented Theodorsen's method ( $-\square-$ and $-\diamond-$ lines) are found to be in good agreement, while discrepancies are reported for high frequency ratios between the actual results and the curves published in the original work [14] (dashed lines with triangles). As already reported by Zeiler [22], the original results, also presented in textbooks [15], may have been affected by numerical errors, probably due to the scarce computational power available at the time. Zeiler's results (Figure 1, * points) are also found to be in good agreement with the actual ones.

\subsection{Results and Observations}

The implemented stability tool has been used to assess the influence of several parameters on a rigid airfoil flutter and divergence limits. Most of the observed trends have already been described in the literature [15, 14], in particular, for low heave-torsion frequency ratios as the case for a HAWT blade section, it is observed that structural damping and elastic axis position scarcely affect the flutter limit, while, on the contrary, the stability limit is very sensitive to other structural parameters as mass, moment of inertia, torsion and heave stiffness.

The sensitivity of flutter limit to the center of gravity position is also confirmed: the more aft the point is located the less stable the section is. As a consequence, a heavy flap device, which increases the airfoil mass in the trailing edge region, generally reduces the flow speed at which flutter occurs. Depending on the structural proprieties of the specific airfoil section and flap, the effects on the stability limits induced by the modified mass distribution might be as significant as the effects of the flap control. 


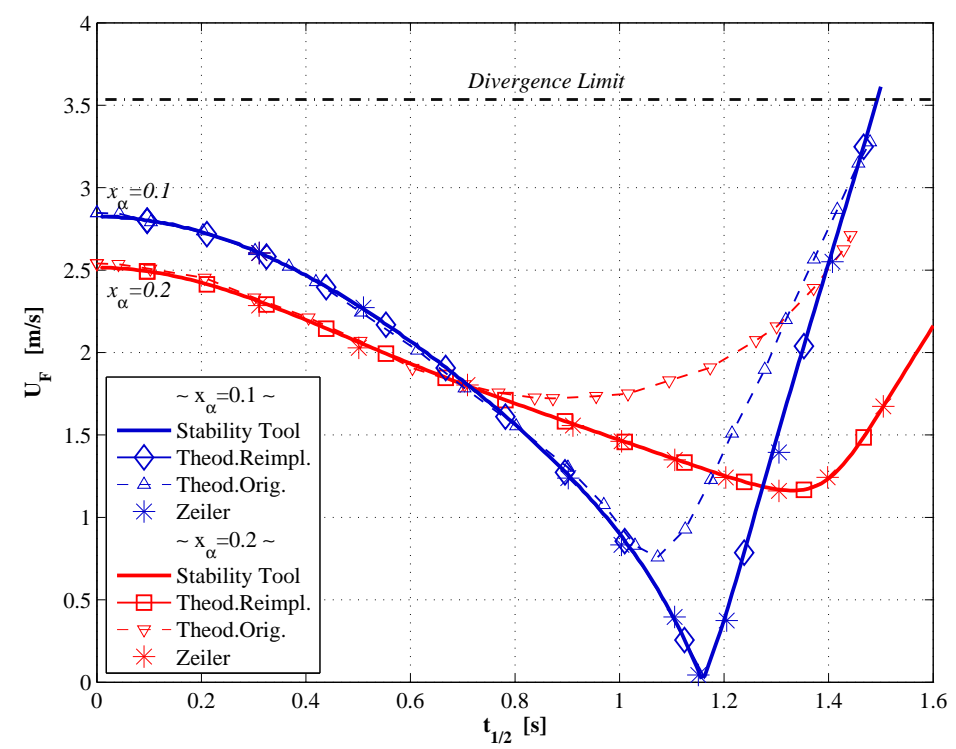

Figure 1: Reduced flutter speed vs. Heave-Torsion frequency ratios: rigid airfoil stability tool validation. Two centre of gravity positions are considered, corresponding respectively to two dimensionless moments of static unbalance: $x_{\alpha}=0.1$ (blue lines with $\diamond$ ) and $x_{\alpha}=0.2$ (red lines with $\square$ ). Flutter curves computed with: actual eigenvalue stability tool (full thick lines), reimplementation of Theodorsen recursive method ( $-\diamond_{-}$and $-\square-$ ). Comparison with: Theodorsen and Garrick (case (q), Graph I-A in [14]) original results (dashed lines with triangles); Zeiler's [22] results (* dots). 
Investigations have been also carried out to determine whether the flutter limit is influenced by the streamwise degree of freedom. It is observed that in case of a flat plate or, generally, symmetric airfoil profiles, the streamwise degree of freedom has no effects on the system stability. If the camber proprieties of the airfoil are instead considered, the mode related to the streamwise DOF may become unstable for lower flow speed. On the other hand, the negative damping introduced by this unstable mode is so low that the streamwise instability completely disappears as a realistic structural damping is applied.

The influence of the streamwise DOF on the flutter limits is hence small in all the investigated cases. It is therefore considered a reasonable assumption to neglect the movements of the structure in the streamwise degree of freedom, obtaining thus a simplified formulation of the aerodynamic model, as will be employed in the model of the flap controlled section.

\section{Model and Method}

The aeroservoelastic model that describes a flap controlled airfoil section consists of structural, aerodynamic and control components. The resulting system of equations is then cast in a state-space matrix formulation and stability, in specified states and flow speed conditions, is investigated by means of eigenvalue analysis. A tool to compute the flutter and divergence limits of an airfoil section equipped with a deformable trailing edge flap control is thereby implemented.

\subsection{Structural Model}

\subsubsection{Reference system}

A local reference system is used to position specific points on the airfoil section. The reference frame (Figure 2, top-right) has origin at the half chord and extends along the airfoil chord with positive direction toward the trailing edge. The dimensionless coordinate $\varepsilon$ is normalized with respect to the half chord length $b_{h c}$ and ranges from -1 , at the leading edge, to +1 , at the trailing edge. The airfoil section is hinged at the point with coordinate $\varepsilon_{e a}$, corresponding to the intersection between the blade elastic axis and the plane where the airfoil section lies. The section centre of gravity, $\varepsilon_{c g}$, is located at a dimensionless distance $x_{\alpha}$ from the elastic axis; $x_{\alpha}$ is usually referred to as dimensionless moment of static unbalance and is positive if the centre of gravity lies aft the elastic axis. The point $\varepsilon_{f l}^{h p}$ where the deformable portion begins is named flap hinge point, in analogy to a 'classic' rigid flap. The center of gravity for the isolated flap is located at the point $\varepsilon_{f l}^{c g}$. 
The mass of the airfoil section is distributed along the chord according to a 'density' function $\rho^{2 D}$. The function $\rho^{2 D}$, in $\mathrm{kg} / \mathrm{m}^{2}$, represents the mass, referred to unit span, of an infinitesimal chord portion of the airfoil section, $b_{h c} \cdot \mathrm{d} \varepsilon$. The total mass of the airfoil section $m_{t o t}$, referred to unit span, is hence the integral of the mass distribution function from the leading to the trailing edge, and, similarly, the moment of inertia for unit span with respect to the elastic axis point $I_{e a}$ is computed as

$$
I_{e a}=\int_{-1}^{+1} \rho^{2 D}\left(\varepsilon-\varepsilon_{e a}\right)^{2} b_{h c}^{2} b_{h c} \mathrm{~d} \varepsilon=I_{c g}+m_{t o t}\left(\varepsilon_{c g}-\varepsilon_{e a}\right)^{2} b_{h c}^{2} .
$$

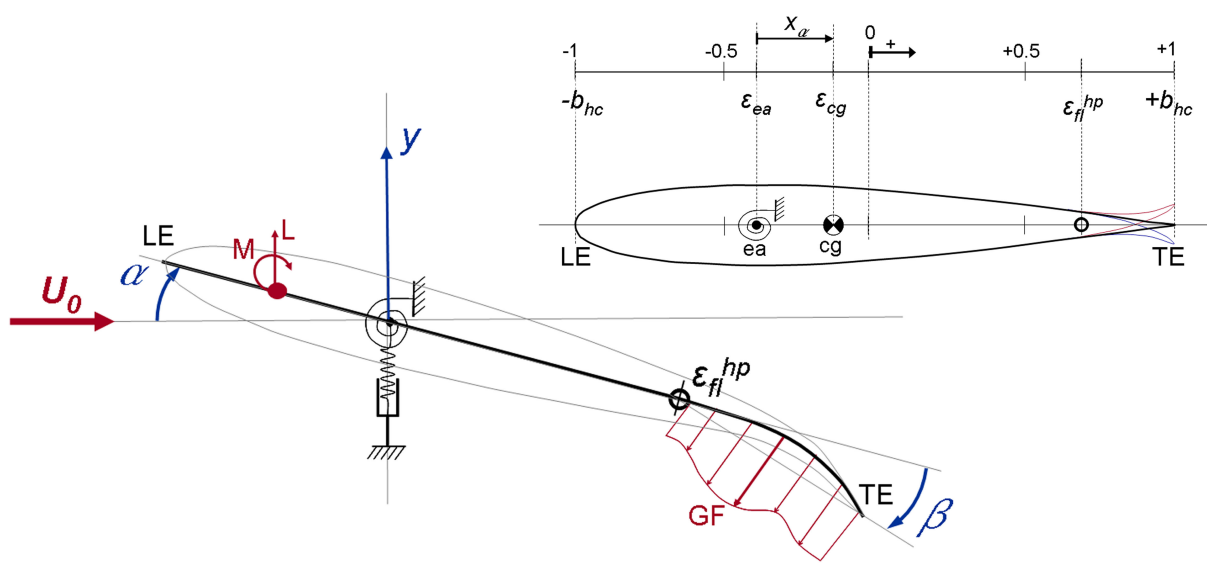

Figure 2: Reference systems. Top-Right: local dimensionless coordinate systems normalized with the half chord length $b_{h c}$. Origin at half chord, positive toward the trailing edge. Bottom-Left: global reference system to define the airfoil motion. Degrees of freedom: heave $(y)$ positive upwards, pitch $(\alpha)$ positive nose up, flap deflection $(\beta)$ positive flap downwards.

The motion and deflection of the flap equipped airfoil are described by three degrees of freedom (Figure 2, bottom-left): $y$ for the rigid body translation in the heave (plunge) direction, $\alpha$ for the rigid rotation that describes the airfoil pitch (torsion) around the elastic axis point $\varepsilon_{e a}$, and $\beta$ to represent the flap deflection. The displacements in the streamwise degree of freedom are neglected since, as concluded in the preliminary study, their influence on flutter or divergence stability limits is small for the case without a flap. The heave coordinate $y$ is positive upwards, and both the pitch $\alpha$ and flap $\beta$ coordinates are considered positive in the clockwise direction, resulting in positive motions when the airfoil pitches nose up and the flap deflects downward. The incoming flow has a constant horizontal direction, parallel to the airfoil resting position, therefore, the structural pitch angle $\alpha$ is also equivalent to the airfoil angle of attack at steady conditions: $\alpha_{\text {Aoa }, s t}=\alpha$. 
The deflection of the trailing edge flap is described by means of a deformation mode shape $u_{f l}$, which defines the shape of the flap by specifying the position of the deformed airfoil camberline when a unit deflection coordinate $\beta$ is assumed, Figure 3. The mode shape is only a function of the chordwise position $\varepsilon$ and it can be arbitrarily scaled. The actual scale factor is chosen so that the coordinate $\beta$ corresponds, fo camberline and $t$ hinge point (dash angle of 1 degree.

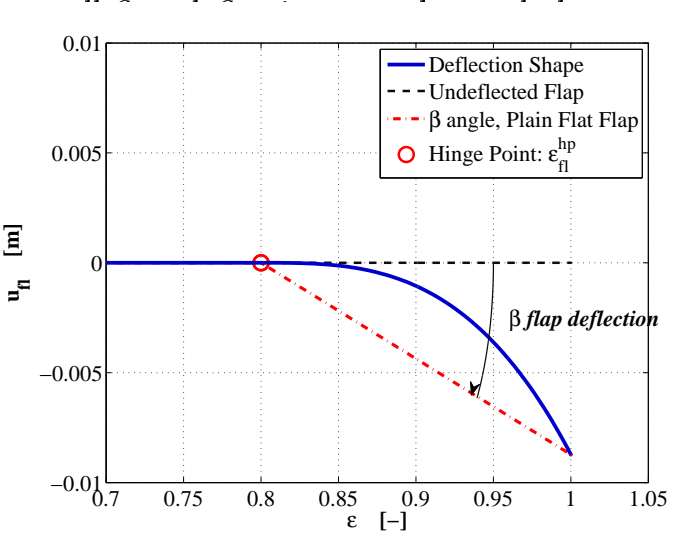
ie undeformed sosition to the esponds to an

Figure 3: Flap deflection mode shape $u_{f l} . \quad \beta=1$ results in $1^{\circ}$ angle between the trailing edge-hinge point line and the undeflected flap position. The plot shows a camberline deformation corresponding to a flap deflection of $\beta=5^{\circ}$. The represented flap deflection mode shape is the one applied to the flap controlled blade section in the following stability analysis and it corresponds to the mode shape employed in [6].

\subsubsection{Equations of motion}

The equations of motion for the section translation and rotation are the classic ones for a suspended rigid body, with the addition of inertial terms related to the flap deflection. Under the assumption of small pitch angles $\alpha$, they read

$$
\begin{array}{ll}
m_{t o t} \ddot{y}-S_{\alpha} \ddot{\alpha}+b_{h c} \text { Ins }_{f l} \ddot{\beta}+c_{y} \dot{y}+k_{y} y & =L_{a e d}, \\
-S_{\alpha} \ddot{y}+I_{e a} \ddot{\alpha}+\left(\varepsilon_{e a} b_{h c}^{2} I_{n s}-b_{h c}^{2} I_{f l} s_{f l}\right) \ddot{\beta}+c_{\alpha} \dot{\alpha}+k_{\alpha} \alpha & =M_{a e d} .
\end{array}
$$

Where, $m_{t o t}$ is the total mass of the airfoil referred to unit span; $S_{\alpha}$ is the moment of static unbalance, $S_{\alpha}=\left(\varepsilon_{c g}-\varepsilon_{e a}\right) b_{h c} m_{t o t} ; I_{e a}$ is the moment of inertia per unit span with respect to the elastic axis, Eq.(1); $c_{d o f}$ is the viscous damping coefficient, $c_{d o f}=2 \xi_{d o f} \omega_{d o f} m_{t o t} ; k_{d o f}$ is the stiffness of the linearelastic springs.

The terms $I n s_{f l}$ and $I m s_{f l}$ represent, as in Gaunaa [12], the contribution 
to the inertial forces from the flap deflection. The terms are defined using a generalized coordinates approach and they correspond to the virtual work carried out by the inertial forces resulting from unit acceleration in the heave and pitch degrees of freedom, respectively; the displacement for the virtual work is then given by the deflection shape. The terms are computed as chordwise integral of the deflection shape $u_{f l}$, the unit-span mass distribution $\rho^{2 D}$ and the chord dimensionless coordinate $\varepsilon$ :

$$
\begin{aligned}
\text { Ins } s_{f l} & =\int_{-1}^{+1} u_{f l} \rho^{2 D} \mathrm{~d} \varepsilon, \\
\text { Ims }_{f l} & =\int_{-1}^{+1} u_{f l} \rho^{2 D} \varepsilon \mathrm{d} \varepsilon .
\end{aligned}
$$

The aerodynamic forcing terms, namely the lift, $L_{a e d}$, and the moment around the hinge point, $M_{a e d}$, are obtained from the aerodynamic model.

The flap equation of motion is derived, in generalized coordinates, from the equilibrium between inertial, damping, elastic and aerodynamic virtual work, computed according to the displacement given by the assigned deflection mode shape:

$b_{h c} \operatorname{Ins}_{f l} \ddot{y}+\left(\varepsilon_{e a} b_{h c}^{2} I n s_{f l}-b_{h c}^{2} I m s_{f l}\right) \ddot{\alpha}+m_{m o d} \ddot{\beta}+c_{f l} \dot{\beta}+k_{f l}\left(\beta-\beta_{c t r l}\right)=G F_{a e d}$,

where, $m_{m o d}$ is the modal mass of the flap deflection mode shape, from the chordwise integral

$$
m_{\text {mod }}=\int_{-1}^{1} u_{f l} \rho^{2 D} u_{f l} b_{h c} \mathrm{~d} \varepsilon
$$

The stiffness of the flap $k_{f l}$ is assigned by setting the natural frequency $\omega_{f l}$ of the deflection mode shape: $k_{f l}=m_{\text {mod }} \omega_{f l}^{2}$. The coupling between the structural model and the control model is given by the term $\beta_{c t r l}$, which represents, as an additional elastic term, the contribution to the flap deflection coming from the control actuator. The generalized force acting on the flap $G F_{\text {aed }}$ is computed as the virtual work of the pressure difference between suction and pressure sides of the airfoil, which is also supplied by the aerodynamic model, just as the lift force $L_{a e d}$ and the pitching moment $M_{a e d}$ :

$$
G F_{a e d}=\int_{-1}^{1} u_{f l} \Delta P b_{h c} \mathrm{~d} \varepsilon .
$$




\subsection{Aerodynamic Model}

The model developed by Gaunaa [12] allows for computation of the aerodynamic forces and their distribution for a 2D airfoil undergoing arbitrary motion and deformation of the camberline. It is thereby possible to describe the aerodynamic forces acting on the flap equipped airfoil. Three main assumptions underlie the formulation: thin airfoil, potential flow and straight line wake. The former assumption implies that the airfoil is represented by its camberline: the thickness is neglected and the airfoil position, motions and deformations are described through the camberline points. The validity of the model is then limited to potential flow condition. Flow separation and stall are not considered and the validity range is restricted to small flap deflections and angles of attack. Furthermore, the effects of the wake on the airfoil are computed assuming that both the airfoil and the wake lie on a straight line. Although included in [12], the streamwise degree of freedom, as previously mentioned, is neglected in the present work. Under these assumptions the aerodynamic model for both integral and distributed (pressure difference over the airfoil) forces is represented by a linear system. The aerodynamic model involves integrals of functions of the deflection mode shapes and their slopes. Since the integrals are evaluated through numerical methods, in order to achieve a sufficient accuracy and avoid biased stability limits, an adequate number of points should be employed to discretize the deflection shape along the chord.

The aerodynamic forces can be split into a non-circulatory and a circulatory contribution. The non-circulatory terms can be interpreted as arising from the inertial forces of the fluid mass in the immediate vicinity of the airfoil being accelerated with the airfoil. They are not affected by wake memory effects and only depend on the instantaneous motion of the airfoil. The circulatory contribution, on the other hand, is linked to the wake vorticity and therefore has a memory effect. This contribution is computed using an effective equivalent down-wash speed $w_{e f f}$, which differs from the correspondent quasi-steady one $w_{3 / 4}$.

The effects of the vorticity shed into the wake are then modeled through an approximation of the indicial response function $\varphi$ as a series of $n_{\text {lag }}$ exponential time lag terms:

$$
\varphi(\tau) \approx 1-\sum_{i=1}^{n_{\text {lag }}} A_{i} e^{-b_{i} \tau}
$$

where, $\tau$ is a dimensionless time variable, $\tau=(1 / b) \int_{0}^{t} U_{r e l} \mathrm{~d} t$, corresponding to the distance, expressed in half-chords, that the airfoil has traveled with respect to the flow. The coefficients $A_{i}$ and $b_{i}$ are parameters that describe the circu- 
latory lift response to a step change in the angle of attack. The coefficients of the two terms approximation for flat plate response computed by Jones [23] are usually adopted. In case of airfoils with a non-zero thickness, a different, and generally slower, response is observed [21], the parameters used to approximate the response of a Ris B1-18 airfoil are reported by Buhl et al. [6], and are listed in Table 1.

Each of the exponential time lag term in the indicial response series is then represented through a first order differential equation,

$$
\dot{z}_{i}=-\frac{1}{b_{h c}} U_{0} b_{i} z_{i}+\frac{1}{b_{h c}} U_{0} b_{i} A_{i} w_{3 / 4}
$$

introducing thus $n_{\text {lag }}$ additional equations and state variables $z_{i}$ in the system.

The indicial response function approximation is then substituted in a Duhamel's superposition integral, and leads to the following expression for the effective equivalent down-wash speed:

$$
w_{\text {eff }}=w_{3 / 4}\left(1-\sum_{i=1}^{n_{\text {lag }}} A_{i}\right)+\sum_{i=1}^{n_{\text {lag }}} z_{i},
$$

where $w_{3 / 4}$ is the quasi-steady equivalent down-wash speed at the three quarter chord point,

$$
w_{3 / 4}=U_{0} \alpha_{A o a, s t}-\frac{1}{2 \pi} U_{0} H_{d y d x} \beta-\dot{y}+b_{h c}\left(0.5-\varepsilon_{e a}\right) \dot{\alpha}-\frac{1}{2 \pi} H_{y} \dot{\beta},
$$

the steady angle of attack $\alpha_{A o a, s t}$ is equal to the structural pitch angle $\alpha$, and $H_{y}$ and $H_{d y d x}$ are integrals of the deflection shape and its slope, as specified in [12].

A full description of the aerodynamic model is reported by Gaunaa [12], together with expression for the aerodynamic forces in the general case of an arbitrary number of deflection shapes and arbitrary motion of these, including motion in the streamwise direction. The expressions, adapted to the current problem using only one deflection shape, lead to the following equations for the unsteady lift force $L_{a e d}$, the pitching moment $M_{a e d}$ and the flap deflection generalized forces $G F_{a e d}$.

$$
\begin{aligned}
L_{a e d}= & \rho b_{h c}^{2} \pi U_{0} \dot{\alpha}+\rho b_{h c}^{2} \frac{1}{\pi} U_{0} F_{d y d x, L E} \dot{\beta} \\
& -\rho b_{h c}^{2} \pi \ddot{y}-\rho b_{h c}^{3} \pi \varepsilon_{e a} \ddot{\alpha}+\rho b_{h c}^{2} \frac{1}{\pi} F_{y, L E} \ddot{\beta} \\
& +2 \rho b_{h c} \pi U_{0} w_{\text {eff }}+L_{c a m b},
\end{aligned}
$$




$$
\begin{aligned}
M_{a e d}= & \rho b_{h c}^{2} U_{0}^{2}\left(\frac{1}{\pi} F_{d y d x, L E}+\frac{1}{2} H_{d y d x}\right) \beta \\
& +\rho b_{h c}^{3} \pi U_{0}\left(0.5-\varepsilon_{e a}\right) \dot{\alpha} \\
& +\rho b_{h c}^{2} U_{0}\left(-\frac{b_{h c}}{\pi} G_{d y d x, L E}+\frac{b_{h c}}{\pi} \varepsilon_{e a} F_{d y d x, L E}+\frac{1}{\pi} F_{y, L E}+\frac{1}{2} H_{y}\right) \dot{\beta} \\
& -\rho b_{h c}^{3} \pi \varepsilon_{e a} \ddot{y}-\rho b_{h c}^{4} \pi\left(1 / 8+\varepsilon_{e a}^{2}\right) \ddot{\alpha}-\rho b_{h c}^{3} \frac{1}{\pi}\left(G_{y, L E}-\varepsilon_{e a} F_{y, L E}\right) \ddot{\beta} \\
& +2 \rho b_{h c}^{2} \pi U_{0}\left(0.5+\varepsilon_{e a}\right) w_{e f f}+M_{c a m b}, \\
G F_{a e d}= & \rho b_{h c} \frac{1}{\pi} U_{0}^{2}\left(P I_{8}-H_{d y d x} P I_{7}\right) \beta \\
& +\rho b_{h c}^{2} U_{0}\left(2 P I_{1}-P I_{6}\right) \dot{\alpha}+\rho b_{h c} \frac{1}{\pi} U_{0}\left(b_{h c} P I_{3}-H_{y} P I_{7}+P I_{9}\right) \dot{\beta} \\
& -2 \rho b_{h c}^{2} P I_{1} \ddot{y}+\rho b_{h c}^{3}\left(-2 \varepsilon_{e a} P I_{1}+P I_{4}\right) \ddot{\alpha}+\rho b_{h c}^{2} \frac{1}{\pi} P I_{2} \ddot{\beta} \\
& -2 \rho b_{h c} U_{0} P I_{5} w_{e f f}+G F_{c a m b} .
\end{aligned}
$$

Where $P I_{n}, F_{\ldots}, f_{\ldots}, G_{\ldots}, H_{\ldots}$ are integrals of the deflection mode shape, as specified in [12]. The integrals are evaluated through numerical methods, therefore a sufficient number of points should be used in the discretization of the flap deflection mode shape along the chord. The terms $L_{c a m b}, M_{c a m b}$ and $G F_{c a m b}$ represent the contribution to the aerodynamic forces that arise with a cambered airfoil profile. The contribution, since the streamwise DOF is neglected, does not depend on the system structural variables and remains constant for fixed free stream flow speeds. The terms have thus no effects on the system stability and can be neglected in the current analysis.

The aerodynamic model also allows to determine the pressure difference between suction and pressure sides of the airfoil in an arbitrary point $\varepsilon_{p}$ on the chord, the pressure difference may then be used as input to flap control algorithms. The pressure difference over the airfoil in case of only one deflection 
mode is given by

$$
\begin{aligned}
\Delta P\left(\varepsilon_{p}\right)= & -\rho \frac{1}{\pi} U_{0}^{2}\left(\frac{\varepsilon_{p}}{\sqrt{1-\varepsilon_{p}^{2}}} H_{d y d x}-\frac{\partial f_{d y d x, \varepsilon_{p}}}{\partial \varepsilon}\right) \beta \\
& +\rho b_{h c} U_{0}\left(2 \sqrt{1-\varepsilon_{p}^{2}}+\frac{\left(2 \varepsilon_{p}+1\right)\left(1-\varepsilon_{p}\right)}{\sqrt{1-\varepsilon_{p}^{2}}}\right) \dot{\alpha} \\
& +\rho \frac{1}{\pi} U_{0}\left(b_{h c} f_{d y d x, \varepsilon_{p}}-\frac{\varepsilon_{p}}{\sqrt{1-\varepsilon_{p}^{2}}} H_{y}+\frac{\partial f_{y, \varepsilon_{p}}}{\partial \varepsilon}\right) \dot{\beta} \\
& -2 \rho b_{h c} \sqrt{1-\varepsilon_{p}^{2}} \ddot{y}+\rho b_{h c}^{2} \sqrt{1-\varepsilon_{p}^{2}}\left(-2 \varepsilon_{e a}+\varepsilon_{p}\right) \ddot{\alpha}+\rho b_{h c} \frac{1}{\pi} f_{y, \varepsilon_{p}} \ddot{\beta} \\
& -2 \rho U_{0} \frac{\varepsilon_{p}-1}{\sqrt{1-\varepsilon_{p}^{2}}} w_{e f f}+\Delta P_{c a m b, \varepsilon_{p}},
\end{aligned}
$$

where the integral functions $f_{y, \varepsilon_{p}}$ and $\partial f_{d y d x, \varepsilon_{p}} / \partial \varepsilon$ are also evaluated in the point of interest $\varepsilon_{p}$ [12]. As mentioned, the contribution from the airfoil profile camber term, $\Delta P_{\text {camb }}$, can be neglected in stability analysis.

The aerodynamic variables are thus available in a linear state-space formulation and the expressions for the aerodynamic forces can be directly substituted in the airfoil equations of motion.

\subsection{Control System Model}

The control system receives as input a set of measurements describing the state of the aeroelastic system and returns as output an angle $\beta_{c t r l}$ that, by changing the deflection of the flap actuator, acts on the same aeroelastic system. The control model consists of a control algorithm part and a time lag one. The control algorithms, according to simple functions of the measured inputs, return a flap deflection $\beta_{\text {set }}$ that would produce the desired changes in the system. In the time lag part then, $\beta_{\text {set }}$ is delayed and substituted by an angle $\beta_{c t r l}$ that actually controls the flap actuator, modeling thus the effects of the time delay that affects the control system, from the acquisition of the sensor inputs to the actuation of the output.

\subsubsection{Control algorithms}

Three different control algorithms are investigated. The first two algorithms are similar to the ones used in Buhl's [6] load alleviation simulations and they are, respectively, based on heave displacement and angle of attack measurements. A third control strategy is then implemented using as input the pressure 
difference between the suction and pressure sides of the airfoil in a specified chordwise location. In this work two chordwise locations will be investigated. The pressure difference control strategy appears particularly interesting due to the simpler sensor set-up required to provide the control inputs; the achievable load alleviation potential is currently under study by Andersen et al. [24].

Heave displacement control The heave control consists of a Proportional Integral Differential (PID) algorithm that aims at reducing the fluctuations of the section heave displacement. The relation between the desired flap deflection and the measured heave displacement and velocity is expressed by the function

$$
\beta_{\text {set }}=A_{y} y+B_{y} \dot{y}-A_{y} y_{I},
$$

where the terms $A_{y}$ and $B_{y}$ are the proportional and differential gain parameters, respectively. The term $y_{I}$ is the heave displacement running mean, computed by integration over a $\Delta T$ window.

The running mean integrand variable can be interpreted as the sum of a constant term and a fluctuating one. The latter term depends on the system state variables and, in stability analysis for specified states of the system, is assumed to oscillate around zero. Furthermore, the oscillations time scale is assumed to be short compared to the integration window $\Delta T$, so that the integral of the fluctuating part tends to be much smaller than the constant term one. As a consequence, the integral corresponding to the running mean term $y_{I}$ can be approximated as constant and hence neglected in the stability analysis. The control algorithm (17) is thus simplified to a linear function in $y$ and $\dot{y}$.

Angle of Attack control The control algorithm attempts to maintain a constant steady lift component by compensating with a flap deflection $\beta_{\text {set }}$ the variation in the angle of attack $\alpha_{A o a, c t r l}$. As in Buhl et al. [6], the control input is computed as a 'quasi-steady' angle of attack:

$$
\alpha_{A o a, c t r l}=\alpha_{A o a, s t}-\frac{1}{U_{0}} \dot{y}+\frac{b_{h c}}{U_{0}}\left(\varepsilon_{A o a, c t r l}-\varepsilon_{e a}\right) \dot{\alpha},
$$

where the steady angle of attack $\alpha_{A o a, s t}$ is equal to the pitch angle $\alpha$. Due to the pitch ratio term $\dot{\alpha}$, the angle of attack input $\alpha_{A o a, c t r l}$ also depends on where the measuring device is located, as expressed by the dimensionless coordinate of the sensor position $\varepsilon_{A o a, c t r l}$.

In the current analysis, the angle of attack sensor is assumed to be located one chord length in front of the leading edge, $\varepsilon_{A o a, c t r l}=\varepsilon_{-2}=-2$, describing, 
for instance, an ideal 5-hole Pitot's tube extending from the blade leading edge [9]. A second sensor location is also investigated, and in this case the measuring point is placed at the 'typical' three quarter chord position: $\varepsilon_{\text {Aoa,ctrl }}=\varepsilon_{3 / 4}=$ 0.5 .

The function relating the measured angle of attack $\alpha_{\text {Aoa,ctrl }}$ to the desired flap deflection $\beta_{\text {set }}$ is then derived from the lift equation (13), simplified by a steady state assumption [6]:

$$
\beta_{\text {set }}=A_{\alpha} \frac{2 \pi}{H_{d y d x}} \alpha_{A o a, c t r l}-A_{\alpha} \frac{2 \pi}{H_{d y d x}} \alpha_{I}+A_{\alpha} \beta_{I},
$$

where, $A_{\alpha}$ is the control gain and the integral terms $\alpha_{I}$ and $\beta_{I}$, under the fast variations assumption, can be neglected in stability analysis.

Pressure difference control In the last algorithm the flap control objective is to maintain a constant pressure difference between the pressure and suction side of the airfoil, at the measurement point. In analogy with the angle of attack control, the control function is derived from the pressure difference equation (16) simplified by a steady state assumption. Neglecting the running mean integral terms, the expression reads

$$
\beta_{\text {set }}=-A_{\Delta P}^{\varepsilon_{p}} \frac{\pi}{2\left(\frac{\partial f_{d y d x, \varepsilon_{p}}}{\partial \varepsilon}-\frac{H_{d y d x}}{\sqrt{1-\varepsilon_{p}^{2}}}\right)\left(0.5 \rho U_{0}^{2}\right)} \cdot \Delta P,
$$

where $A_{\Delta P}$ is the control gain, $\varepsilon_{p}$ is the location of the pressure measurement point and $\partial f_{d y d x} / \partial \varepsilon$ is a deflection shape integral evaluated at the measurement point $\varepsilon_{p}$ [12]. Two different measurement point locations will be employed in the following. The first point, $\varepsilon_{c n s t}=-0.029$, is close to the airfoil half chord and in static conditions, the ratio between the lift coefficient and the pressure coefficient at this specific point is constant [20]. This means that for quasi steady conditions, keeping the pressure difference constant in this point results in a constant lift. The second pressure difference measurement point is located at $10 \%$ chord from the leading edge, $\varepsilon_{10 \%}=-0.8$, because first simulations [24] have shown more promising load alleviation potentiality with pressure sensors in the fore part of the airfoil.

\subsubsection{Control time lag}

The time lag that may affect the control system is modeled as a first order filter that delays the algorithm output $\beta_{\text {set }}$ and returns the deflection angle $\beta_{c t r l}$, then applied to the flap actuator. The delay function is formulated in state-space as 
a first order differential equation,

$$
\dot{\beta}_{c t r l}=\frac{\ln (0.5)}{t_{1 / 2}} \beta_{c t r l}-\frac{\ln (0.5)}{t_{1 / 2}} \beta_{\text {set }},
$$

The reaction half time, $t_{1 / 2}$, represents the control lag as the time required by the control output $\beta_{\text {ctrl }}$ to reach half of its final value $\beta_{\text {set }}$ following a step input. The equation grows stiffer as the time lag is reduced, therefore, in the ideal case of no time lag, equation (21) is not applicable and the delay function simply reduces to $\beta_{\text {ctrl }}=\beta_{\text {set }}$.

\subsection{Aeroservoelastic System. Eigenvalue Solution}

The equations describing the aeroservoelastic system are all linear in the system state variables. By introducing a variable substitution for the second order derivatives of the structural variables, the full set of equations can be cast in a linear matrix equation of the first order:

$$
M \underline{\dot{x}}=A \underline{x}+\underline{f_{0}} .
$$

Where, $\underline{x}$ is a vector that collects all the aerodynamic, control and structural variables of the aeroservoelastic system. As an example, in case of a three terms indicial response approximation and time lag in the control, the system would involve 10 first order differential equations: 3 for variable substitution, 3 equations of motion, 3 aerodynamic wake memory terms equations and 1 for the control time lag model. The system variables vector would hence be

$$
\underline{\boldsymbol{x}}=\left\{\dot{y}, \dot{\alpha}, \dot{\beta}, y, \alpha, \beta, z_{1}, z_{2}, z_{3}, \beta_{c t r l}\right\} .
$$

The behavior of the airfoil structure can be simulated in the time domain by numerically integrating the set of aeroservoelastic equations through a time marching algorithm. The resulting response history of the state variable vector $\underline{\boldsymbol{x}}_{(t)}$ would then indicate whether the system in the specified conditions is stable or not.

On the other hand, if the system is linear or linearized, stability analysis is more conveniently carried out in the frequency domain using an eigenvalue approach [19]. A solution in the harmonic form $\underline{\boldsymbol{x}}_{(t)}=\underline{\phi}^{\lambda t}$ is assumed for the matrix equation (22), leading to the generalized eigenvalue problem

$$
(\boldsymbol{A}-\lambda \boldsymbol{M}) \underline{\phi}=\underline{\mathbf{0}} .
$$

The eigenproblem solution consists in a set of eigenvalues $\lambda_{j}$ and respective 
eigenvectors $\underline{\phi}_{j}$, which characterize the modes describing the aeroservoelastic system. The imaginary part of each eigenvalue $\operatorname{Im}\left(\lambda_{j}\right)$ returns the frequency of the mode, in rad/s, while the real part $\operatorname{Re}\left(\lambda_{j}\right)$ relates to the modal damping. A negative eigenvalue real part corresponds to a stable positively damped modal contribution and the mode logarithmic damping can be computed as

$$
\delta_{j}=-\frac{\operatorname{Re}\left(\lambda_{j}\right)}{\operatorname{Im}\left(\lambda_{j}\right)} .
$$

The stability limits are then determined in the frequency domain by solving the eigenproblem for increasing flow speed $U_{0}$. The aeroservoelastic system in a specified condition is stable only if all the modes are positively damped, therefore, the flow speed at which any of the modes turns negatively damped indicates the stability limit. If the mode turning unstable has a null frequency $\operatorname{Im}(\lambda)=0$, then divergence (static instability) occurs, otherwise, a negatively damped mode with a positive frequency $\operatorname{Im}(\lambda)>0$ yields to a flutter instability. The system modes, computed at different flow speeds, are then tracked and identified by means of a modal assurance criterion [25] based on the eigenvector modal shapes.

\section{Validation}

The actual model and its implementation are validated against flutter curves computed with a reimplementation of Theodorsen's [13] recursive method for a three degrees of freedom flutter problem. The system considered by Theodorsen [13] and by Theodorsen and Garrick [14] consists in a flat plain flap hinged to the aft edge of a rigid flat plate. The plate translates in the heave direction and rotates around its elastic axis. The rigid flap rotates around its hinge point connected to the plate, linear springs constrain the movements in all the three degrees of freedom and no control is applied.

In order to investigate Theodorsen's system with the actual stability tool, a linear deflection mode shape is defined to represent the rigid flap rotation:

$$
u_{f l}=\left\{\begin{array}{cl}
-\left(\varepsilon-\varepsilon_{f l}^{h p}\right) b_{h c} & \text { if } \varepsilon>\varepsilon_{f l}^{h p}, \\
0 & \text { otherwise. }
\end{array}\right.
$$

The terms $I m s_{f l}(5), \operatorname{Ins}_{f l}(4)$, and $m_{m o d}(7)$ are then evaluated according to 
the flat flap deformation shape (26):

$$
\begin{aligned}
m_{\text {mod }} & =I_{f l, h p}=m_{t o t} b_{h c}^{2} r_{\beta}^{2}, \\
I n s_{f l} & =-m_{f l a p}\left(\varepsilon_{f l}^{c g}-\varepsilon_{f l}^{h p}\right)=-m_{t o t} x_{\beta}, \\
I m s_{f l} & =-\frac{1}{b_{h c}^{2}}\left(I_{f l, h p}-I n s_{f l} \varepsilon_{f l}^{h p} b_{h c}\right),
\end{aligned}
$$

where, $I_{f l, h p}$ is the moment of inertia with respect to the hinge point of the isolated flap and $m_{\text {flap }}$ its total mass. The terms $r_{\beta}$ and $x_{\beta}$ refer to dimensionless parameters for, respectively, the moment of inertia and the flap center of gravity position, defined as in the previous works $[13,14]$. Furthermore, in order to specify the indicial response function for the flat plate airfoil, the current tool employs a three terms approximation, Eq. (9). The $A_{i}$ and $b_{i}$ parameters that define the response function have been computed by seeking the best fitting [21] between the indicial curve from the approximated expression and the corresponding curve obtained from an unsteady lumped-vortex panel code [26]. A response function based on the classic two terms approximation by Jones [23] is also used as comparison. ${ }^{1}$

Flutter limits are then computed for the flap equipped section described in Theodorsen and Garrick (Figure 8 in [14]), the resulting flutter speeds are plotted (Figure 4) versus the ratio between flap deflection and torsion natural frequencies $\omega_{f l} / \omega_{\alpha}$; as in [14], the heave-torsion frequency ratio is maintained constant: $\omega_{y} / \omega_{\alpha}=0.2$. Results from the actual stability tool (Figure 4 , thick full line) are compared to the ones obtained with the reimplementation of Theodorsen's recursive method (- $\diamond-$ line), the original results [14] are also displayed $(-\triangle-$ line $)$.

The curves obtained with the two methods are in good agreement and the stability tool can be considered validated. Further validation tests, including cases with controlled flap, are carried out against time marching solutions. As also observed in the two degrees of freedom flutter problem addressed in the preliminary study, the original flutter curves reported by Theodorsen and Garrick [14] are biased, probably due to the limited computational means available at that time.

The effects of the wake vorticity on the flat plate aerodynamic are modeled through analytical functions in Theodorsen's recursive method, while an indicial response function approximation is adopted in the stability tool, resulting thus in small differences between the respective flutter curves. It is also observed

\footnotetext{
${ }^{1}$ The coefficients for the indicial response function approximation using a three terms exponential series are $A_{1}=0.0182, A_{2}=0.2411, A_{3}=0.2407$, and $b_{1}=3.02 \cdot 10^{-6}, b_{2}=$ $0.3989, b_{3}=0.0818$. Jones coefficients are $A_{1}=0.1650, A_{2}=0.3350$, and $b_{1}=0.0455, b_{2}=$ 0.3000
} 


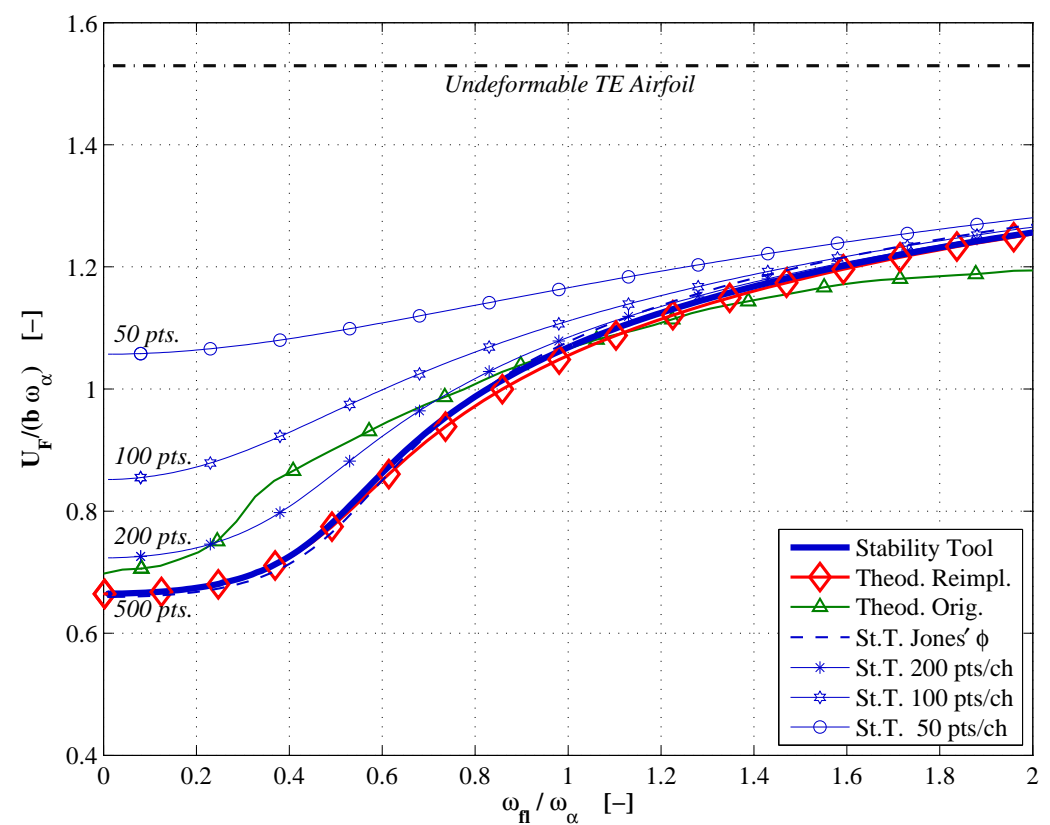

Figure 4: Stability tool validation. Reduced flutter speed vs. Flap-Torsion frequency ratio, constant $\omega_{y} / \omega_{\alpha}=0.2$. Flat plate equipped with a rigid rotating undeflected flap. Comparison of flutter limits obtained with: current stability tool (thick full line), reimplementation of Theodorsen's recursive method $\left(-\diamond_{-}\right)$, original results from Figure 8 in [14] $(-\triangle-)$. Indicial response function approximations: 2 terms Jones' (dashed line) and 3 terms (thick full line). Convergence analysis, number of points to discretize the deflection shape along the chord: current stability tool, 500 pts (thick full line); 200 pts (stars); 100 pts (hexagons); 50 pts (circles). 
that the curve based on Jones' two term approximation (Figure 4, dashed line), although sufficiently close to the analytical solution ( - - line), appears slightly less precise than the one obtained with a three terms response approximation (thick full line).

As described in the previous section, the aeroelastic system is modeled through a set of equations that include integral functions of the deflection shape along the chord. The deflection shape integrations are carried out with numerical methods, therefore, the accuracy of the terms, and thereby of the computed stability limits, is proportional to the number of points used to discretize the mode shape along the chord. A convergence analysis is hence required to determine the adequate number of discretization points.

In the actual validation case (Figure 4), a sufficient accuracy is achieved with a 500 points grid. The differences from more refined discretization are negligible, whereas biased flutter curves (200, 100 and 50 points lines in Figure 4) are observed with a lower number of points. In the following blade section investigation, a larger number of points (2000, as in [20]), is instead required to discretize with sufficient accuracy the non linear deflection mode shape (Figure 3) of the deformable trailing edge flap.

\section{Stability of a Flap Controlled Blade Section}

The implemented stability tool is now applied to investigate the flutter and divergence limits of an airfoil equipped with a deformable trailing edge control. The investigated airfoil section is identical to the one adopted in the load alleviation simulations from Buhl et al. [6] and the section structural proprieties are set to be representative of a MW-size blade section with a Ris B1-18 airfoil profile. Stability limits of a blade section actively controlled for fatigue load alleviation are thus determined and the effects of the deformable flap on stability are assessed.

The structural properties of the investigated airfoil are reported in Table 1, together with the three pairs of parameters that describe the indicial response function approximation for the Ris B1-18 airfoil profile [6]. The control gain parameters (Table 1) are tuned for fatigue load alleviation at a flow speed close to $60 \mathrm{~m} / \mathrm{s}$. Gain parameters from the optimization in [6] are adopted for the heave and angle of attack algorithms, while the two versions of the pressure difference controls are tuned with Ziegler-Nichols' method [27], since optimized parameters were not yet available at the time writing.

Results indicate that the flap control significantly modifies the stability limits of the airfoil section. In fact, the flow speed at which flutter occurs on a flap equipped section differs from the rigid airfoil one and it varies in a non-trivial 


\begin{tabular}{|l|c|cc||cc|}
\hline \multicolumn{5}{|c||}{ Airfoil structural proprieties } & \multicolumn{2}{c|}{ Indicial Response Approximation } \\
Ris B1-18 Airfoil Profile, 3 terms:
\end{tabular}

Table 1: Characteristics of the investigated reference airfoil section, same as in [6]. Structural proprieties for airfoil and flap. Indicial response approximation terms for a Ris B1-18 airfoil profile. Control gain for fatigue load alleviation at $60 \mathrm{~m} / \mathrm{s}$.

fashion depending on several parameters: flap stiffness, mass distribution, control algorithm, time lag and gain values. First, results are presented for the case of a deformable trailing edge flap without any control acting on it (undeflected elastic flap), subsequently, the stability curves obtained with the described control systems are displayed (controlled elastic flap).

\subsection{Undeflected Elastic Flap}

The presence of the deformable trailing edge flap modifies the flutter limit of the airfoil section even when the flap is not deflected by the control system. The resulting flutter limits are then depending on the flap structural characteristics, namely its stiffness and mass distribution.

For a reasonably stiff flap, as the one installed on the investigated reference section, the flow velocity at which flutter sets in is actually increased compared to the case with a completely rigid airfoil (Figure 5, thick full line). As the flap stiffness is increased, flutter speed converges to the limit computed with the preliminary model of a rigid airfoil without flap. On the other hand, if an excessively soft flap is mounted, flutter could occur at much lower flow speeds. In this case, the mode related to the flap deflection is responsible for the instability, while the stabilizing effects on the remaining modes are still present (Figure 5, dashed line). The flutter curve in this region has a nearly linear trend and its slope depends on the flap mass distribution. In fact, a milder slope, and thus a wider low stability region, is observed in case of flaps with larger mass or more aft centre of gravity [20].

The flap equipped blade section is then investigated to assess how different approximations in the aerodynamic model may influence the stability limits predictions, Figure 5. A common approximation simplifies the unsteady aero- 


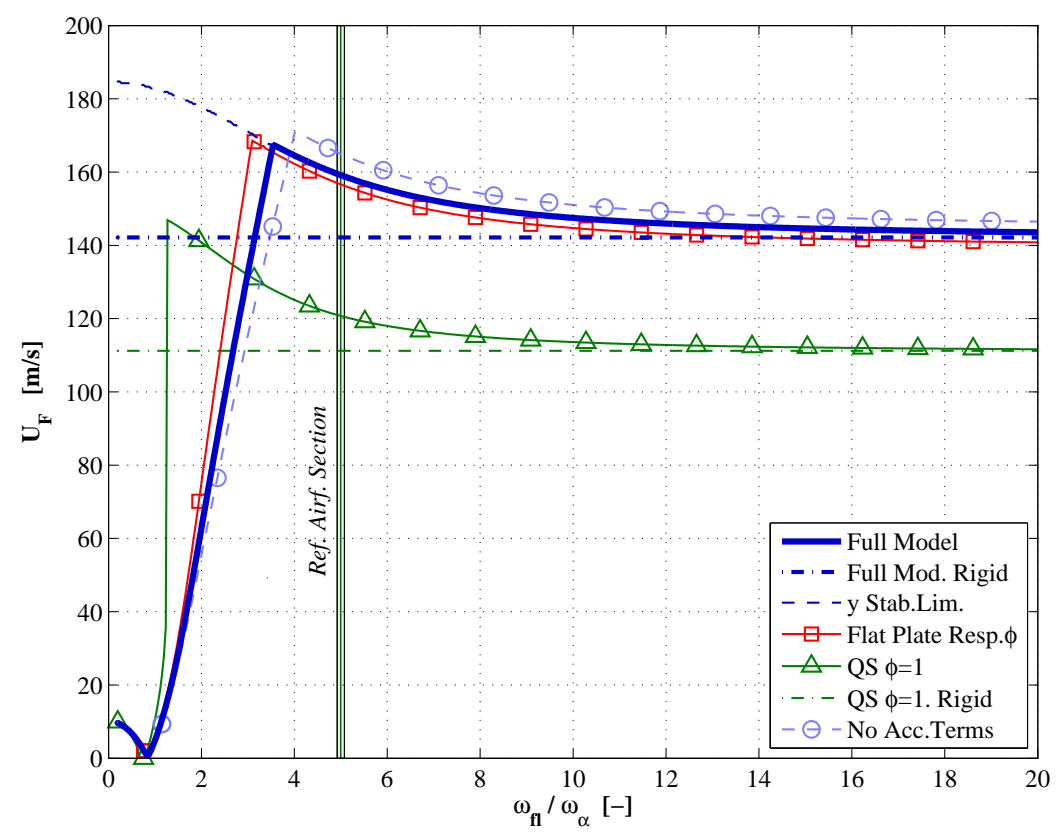

Figure 5: Flutter flow speed vs. Flap-Torsion natural frequency ratios. Airfoil equipped with undeflected deformable trailing edge (full thick line). Rigid airfoil (dash-and-dot line). The flap-torsion frequency ratio for the reference airfoil section, $\omega_{f l} / \omega_{\alpha}=5$, is indicated by the vertical line. Investigation on different aerodynamic model approximations: stability tool full model (full thick line); quasi-steady approximation $(-\triangle-)$; Jones' approximation for flat plate response $(-\square-)$; neglect acceleration terms in the aerodynamic forces (-०-). 
dynamic model to a Quasi-Steady one by neglecting the effects of the vorticity shed into the wake. The response function $\varphi$ is thus constant and equal to unity, and the effective equivalent down-wash speed $w_{\text {eff }}$, Eq. (11), simply reduces to the quasi-steady equivalent down-wash $w_{3 / 4}$, Eq. (12). The resulting curve $(-\triangle-$ line) largely underestimates the section flutter limit for most of the frequency ratios, as also observed on a rigid airfoil by Hansen [18] and on a full blade by Lobitz [17]. It is therefore concluded that the wake vorticity has a stabilizing effect that can not be neglected in a stability analysis.

The full model curve is computed considering an indicial function approximation of the Ris B1-18 response. The coefficients for a three terms series approximation are given in Buhl et al. [6] and here reported in Table 1. Hansen et al. [21] observe that an airfoil with finite thickness, such as the Ris B1-18, has a different, and generally slower, indicial response than the flat plate one. The difference in response then influences the corresponding stability curves. In fact, the flutter limits computed with Jones' coefficients for a flat plate (Figure $5,-\square-$ line) deviate from the full model curve with a Ris B1-18 airfoil. Although not negligible, the difference between the two stability curves is rather small. Therefore, in case the response for the specific airfoil is unknown, the flat plate approximation can be considered a reasonable assumption for flutter analysis.

It is also observed that neglecting the contributions to the aerodynamic forces from the acceleration terms $(\ddot{y}, \ddot{\alpha}, \ddot{\beta})$ results in only slightly overestimated flutter limits (-o- line), whereas the $U_{0} \dot{\alpha}$ contribution to the non circulatory forces is necessary to avoid completely biased stability limits.

\subsection{Controlled Flap}

The deformable trailing edge flap is now controlled for fatigue load alleviation according to the described control algorithms, and the ideal case of a null control time lag is considered at first (Figure 6). Each control algorithm results in a diverse stability curve, but all of them present the same qualitative trend already observed in the undeflected flap case (full thick line): in an initial range of frequency ratios, the flap deflection mode is responsible for the instability and the flutter limit increases almost linearly with the flap stiffness up to a maximum point; for higher flap frequencies, the flutter limit slightly decreases and approaches an horizontal asymptote.

Whenever the reference section (vertical line) is actively controlled for fatigue load alleviation, it undergoes flutter at flow speeds lower than the undeflected flap case. A decreased stability limit is reported with any of the control algorithms, and, with most of them, the flutter limit is also below the rigid airfoil one. Nevertheless, in the investigated cases, a critically low limit is only re- 


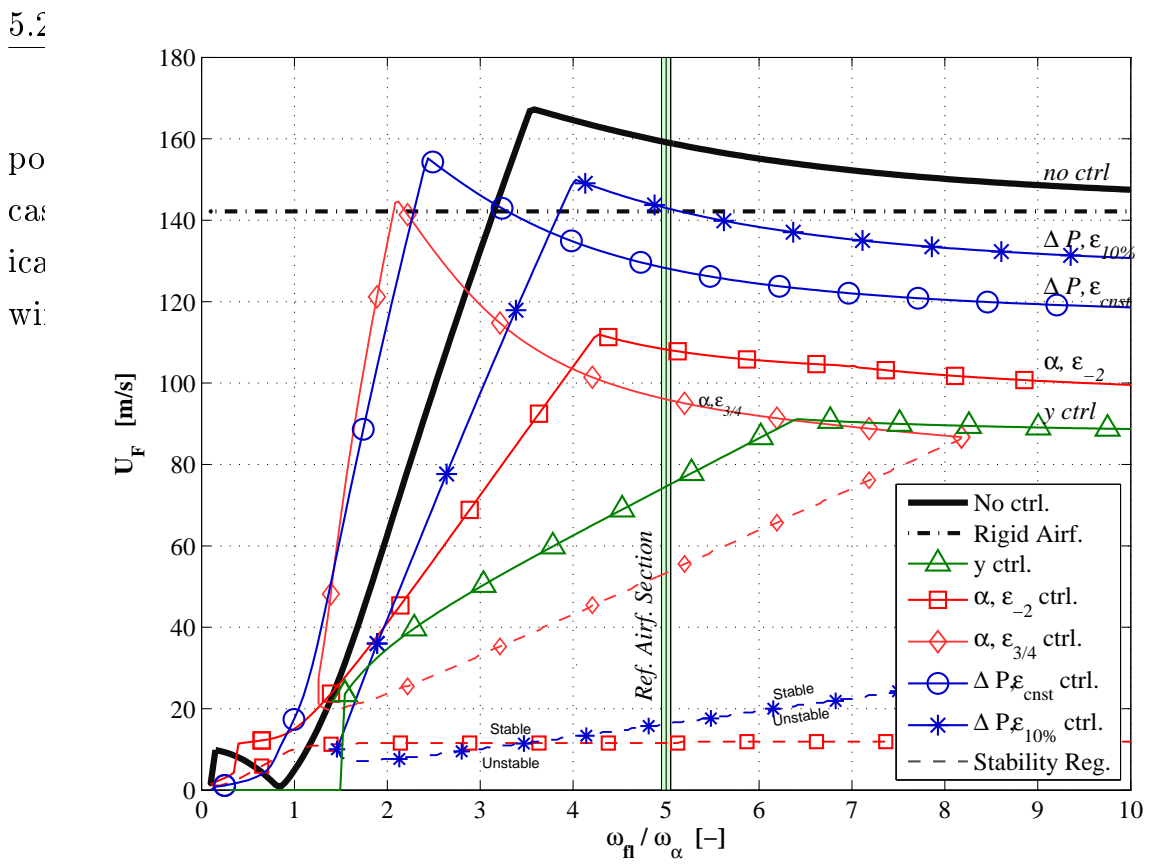

Figure 6: Flutter flow speed vs. Flap-Torsion natural frequencies ratios for the different control algorithms. Reference section frequency ratio $\omega_{f l} / \omega_{\alpha}=5$ at the vertical line. Deformable trailing edge flap controlled for fatigue load alleviation, null control delay assumed. Control algorithms: heave $(-\triangle-)$; angle of attack, measured at $\varepsilon_{-2}(-\square-)$ and at $\varepsilon_{3 / 4}\left(-\diamond_{-}\right)$; pressure difference at $\varepsilon_{\text {cnst }}(-\circ-)$ and at $\varepsilon_{10 \%}(-*-)$. Stability regions are reported for the angle of attack and pressure difference algorithms: the system is stable for flow speeds from the dashed line to the full one. Undeflected flap flutter curve (full thick line) and the rigid trailing edge airfoil one (dash-and-dot) are displayed for comparison.

The stability limits are thus depending on the type of algorithm used to control the trailing edge flap, but also on the location of the measurement device that provides the control input. For instance, in the angle of attack case, the same control algorithm returns rather different curves considering the two measurement locations. While the $\varepsilon_{-2}$ curve shows an ordinary trend, in the $\varepsilon_{3 / 4}$ case, the instability region observed at low flow speeds grows as the flap stiffness increases, till the stability region is occluded and, for higher frequency ratios, the system get unstable in the whole flow speed range.

A dependency of the stability limits on the control gain parameters is also reported. In fact, by decreasing the gain magnitudes, stability limits increase and the flutter curves approach the one obtained with the undeflected flap (full 
thick line), which can be interpreted as a particular control with all the gain parameters set to zero.

Stability limits even above the undeflected flap case can be achieved with a dedicated re-tuning of the gain parameters [20], thus obtaining an active suppression of the aeroelastic instabilities through the flap control. On the other hand, active flutter suppression probably has little interest for wind turbine applications. In fact, the investigated section presents an undeflected flap flutter limit already above the rigid airfoil one. Therefore, in the event of a wind turbine rotor over-speeding and unusually high flow speeds, flutter could be avoided, or at least postponed, by simply excluding the control system and consequently rise the stability limit to the undeflected flap one. Particular precautions should be taken in case the exclusion or a failure in the control system would also compromise the flap stiffness, for instance in case of a pneumatically actuated flap.

The flow speed limits at which static instabilities as divergence occur are also observed to vary depending on the flap control characteristics. Nonetheless, in all the investigated cases, flutter occurs at much lower flow speeds than divergence does. The stability limits observed on the investigated flap equipped section are thus referable exclusively to flutter instabilities.

Control Time Lag In previous studies [6, 28] it was clearly outlined how by introducing time lag in the control system the load alleviation achievable with the flap control would distinctly decrease as the control delay increases. The control time lag also affect the section stability limits, but its effects are indeed not as clearly defined (Figure 7). In fact, in the investigated case, if the control time lag, quantified through the half time $t_{1 / 2}$, is increased, the flutter limit for the $\Delta P_{\varepsilon, 10 \%}$ algorithm slightly increases (-*- line), and almost no effects are reported for the angle of attack case $(-\square-)$. Whereas, a less homogeneous response is observed whenever the flap is actuated according to the $\Delta P_{\varepsilon, \text { cnst }}$ pressure difference algorithm (-o-) or to the heave one $\left(-\triangle^{-}\right)$. In the latter case, the flutter limit initially rises up to the control reversal speed $(95.5 \mathrm{~m} / \mathrm{s}$ for the considered section) and then plunges below $10 \mathrm{~m} / \mathrm{s}$. The $\Delta P_{\varepsilon, \text { cnst }}$ pressure control algorithm is completely unstable for small time lag, while for longer control delays the section is only stable inside a region, from the dashed line to the full one; stability regions are also reported in the angle of attack control cases. 


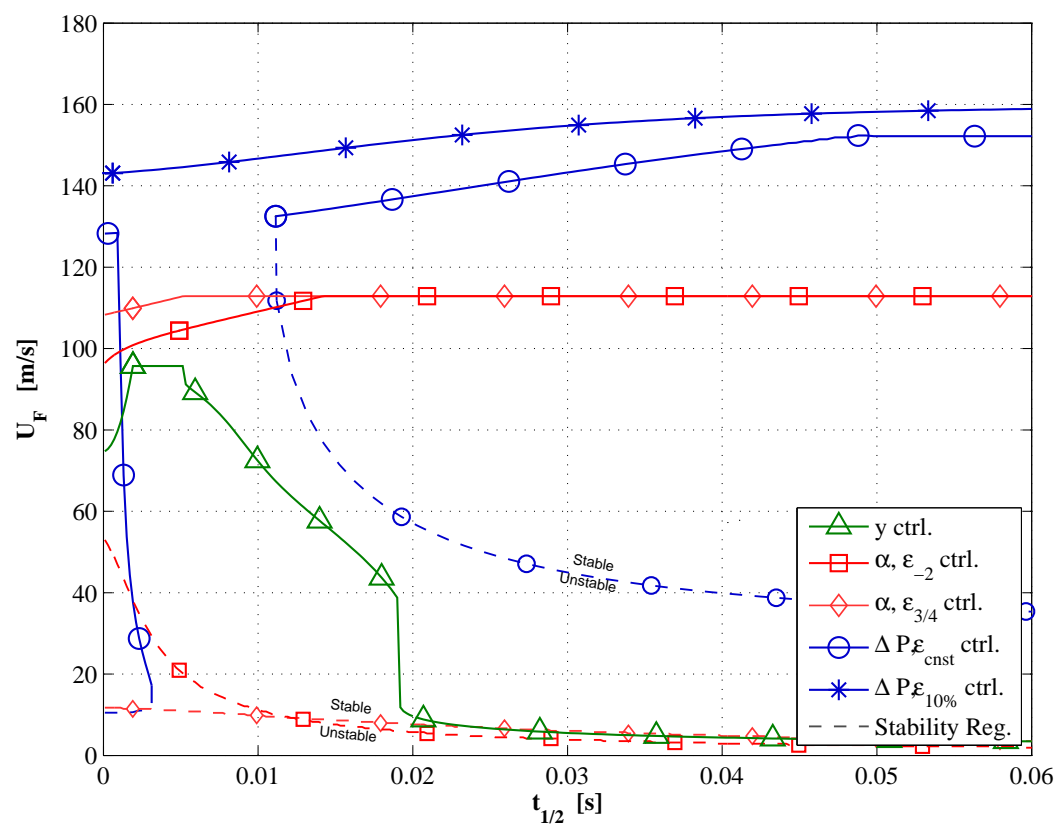

Figure 7: Flutter flow speed vs. control time lag, quantified by the control reaction half time $t_{1 / 2}$. Reference section frequency ratio $\omega_{f l} / \omega_{\alpha}=5$ at the vertical line. Deformable trailing edge flap controlled for fatigue load alleviation. Control algorithms: heave $(-\triangle-)$; angle of attack, measured at $\varepsilon_{-2}(-\square-)$ and at $\varepsilon_{3 / 4}(-\diamond)$; pressure difference at $\varepsilon_{\text {cnst }}(-$ $\circ-)$ and at $\varepsilon_{10 \%}(-*-)$. Stability regions are reported for the angle of attack and pressure difference algorithms: the system is stable for flow speeds from the dashed line to the full one. 


\section{Conclusion}

A model to determine the flutter and divergence limits of a 2D airfoil section equipped with a deformable trailing edge flap control is proposed and implemented in a stability tool. The tool is validated against time marching solutions and against the stability limits computed with a reimplementation of Theodorsen's [13] recursive method. The stability limits computed with the implemented tool and the recursive method results are in excellent agreement, while the flutter curves presented in Theodorsen and Garrick [14] are found to be biased. This is thought to be caused by the limited computational power and accuracy available at the time the original work was carried out.

Flutter and divergence speeds are then computed for a wind turbine blade section where a flap control is applied to actively alleviate fatigue loads, as in [6]. It is thereby observed that the presence of the deformable trailing edge flap, either actively controlled or not, significantly modifies the section stability limits. In case of an undeflected elastic flap, not manoeuvred by any control, the stability limits depend on the flap structural properties. Soft and heavy flaps result in lower flutter speeds, while a reasonably stiff flap, as the one considered in the investigated reference airfoil case, actually increases the velocity limit at which flutter occurs to a value above the rigid airfoil one. As observed in the preliminary study, the presence of the flap also alters the mass distribution of the whole airfoil section. In fact, the trailing edge flap structure might increase the weight of the aft part of the airfoil and, consequently, reduce the flow speed at which flutter occurs. Depending on the structural proprieties of the specific airfoil section and flap, the effects on the stability limits induced by the modified mass distribution might be as significant as the effects of the flap control.

By activating the control algorithms for fatigue load alleviation, the dependency of the stability limits on the flap structure remains, and lower limits are reported, in most of the cases below the rigid airfoil flutter speed. However, only the flap control based on heave displacement returns a flutter speed as low as half the rigid airfoil one, setting thus a stability limit critically close to the flow speeds encountered by a wind turbine blade in normal operation. Rather good performances from a stability point of view are instead reported for the pressure control algorithm, especially in the case of pressure difference measurement located at the $10 \%$ chord position. In any case, the undeflected flap airfoil section has a higher stability limit than the rigid airfoil, which renders the control system 'fail-safe' for stability. In fact, in the event of control failure or rotor over-speeding, a rather high stability limit is set by simply excluding the control (if the event does not have any repercussion on the flap stiffness).

Stability limits are found to depend also on the time lag in the control 
algorithm, although a clear common trend is not recognizable and different effects are observed for each control algorithm.

The implemented stability tool allows to determine the effects of the flap control on the stability of an airfoil $2 \mathrm{D}$ section. The transition from a $2 \mathrm{D}$ section to a rotating full blade 3D case is not trivial. Finite span aerodynamic, blade rotation, higher order deflection modes, structure and control variations along the blade span are all affecting the response of the complete 3D blade, and consequently, stability limits for a 3D case can not be easily extrapolated from 2D investigations. Nevertheless, a 2D analysis allows to capture the underlying stability behavior of a blade section and highlights that the flap control has important effects on the airfoil aeroservoelastic stability. The flow speed at which flutter or divergence occur on a flap controlled section then varies depending on several factors: the airfoil and flap structural proprieties, the type of control algorithm, the control inputs, the gain parameters and the time lag in the control.

To conclude, the effects of a flap control on the system stability are certainly neither negligible nor straightforward. Therefore, the usability of a stability tool able to report such effects appears to be significant.

\section{Acknowledgment}

This work is partly sponsored by the Danish National Advanced Technology Foundation through the project 'Adaptive Trailing Edge Flaps for Wind Turbines'.

\section{References}

[1] E.A. Bossanyi. Individual blade pitch control for load reduction. Wind Energy, 6(2):119-128, 2003.

[2] T.J. Larsen, H.A. Madsen, and K. Thomsen. Active load reduction using individual pitch, based on local blade flow measurements. Wind Energy, 8(1):67-80, 2005.

[3] T.K. Barlas and G. van Kuik. State of the art and prospectives of smart rotor control for wind turbines. Journal of Physics: Conference Series, 75(1):012080 (20 pp.), 2007.

[4] D.T.Y. Nakafuji, C.P. van Dam, R.L. Smith, and S.D. Collins. Active load control for airfoils using microtabs. Transactions of the ASME. Journal of Solar Energy Engineering, 123(4):282-289, 2001. 
[5] C.P. van Dam, R. Chow, J.R. Zayas, and D.E. Berg. Computational investigations of small deploying tabs and flaps for aerodynamic load control. Journal of Physics: Conference Series, 75(1):012027-1-10, 2007.

[6] T. Buhl, M. Gaunaa, and C. Bak. Potential load reduction using airfoils with variable trailing edge geometry. Transactions of the ASME. Journal of Solar Energy Engineering, 127(4):503-16, 2005.

[7] N. Troldborg. Computational study of the ris-b1-18 airfoil with a hinged flap providing variable trailing edge geometry. Wind Engineering, 29(2):89113, 2005 .

[8] P.B. Andersen, M. Gaunaa, C. Bak, and M.H. Hansen. A dynamic stall model for airfoils with deformable trailing edges. Journal of Physics: Conference Series, 75(1):012028-1-10, 2007. Paper submitted for publication to Wind Energy.

[9] P.B. Andersen, L.C. Henriksen, M. Gaunaa, C. Bak, and T. Buhl. Integrating deformable trailing edge geometry in modern mega-watt wind turbine controllers. In Scientific proceedings. 2008 European Wind Energy Conference and Exhibition, Brussels (BE), pages 15-21, 2008. Paper submitted for publication to Wind Energy.

[10] J.W. van Wingerden, A.W. Hulskamp, T. Barlas, B. Marrant, G.A.M. van Kuik, D.P. Molenaar, and M. Verhaegen. On the proof of concept of a 'Smart' wind turbine rotor blade for load alleviation. Wind Energy, 11(3):265-280, 2008.

[11] Vasilis A. Riziotis and Spyros G. Voutsinas. Aeroelastic modeling of the active flap concept for load control. In Scientific proceedings. 2008 European Wind Energy Conference and Exhibition, Brussels (BE), pages 22-26, 2008.

[12] Mac Gaunaa. Unsteady 2D potential-flow forces on a thin variable geometry airfoil undergoing arbitrary motion. Technical Report 1478, Ris National Laboratory, Roskilde (DK), 2006. Paper submitted for publication to Wind Energy.

[13] T. Theodorsen. General theory of aerodynamic instability and mechanism of flutter. Technical Report 496, National Advisory Committee for Aeronautics (United States Advisory Committee for Aeronautics), 1935.

[14] T. Theodorsen and I.E. Garrick. Mechanism of flutter, theoretical and experimental investigation of flutter problem. Technical Report 685, National Advisory Committee for Aeronautics (United States Advisory Committee for Aeronautics), 1940. 
[15] Raymond L. Bisplinghoff, Holt Ashley, and Robert L. Halfman. Aeroelasticity. Dover Pubblications, Inc, 1996.

[16] Mac Gaunaa. Investigation of the effect of deformable trailing edge geometry control systems on flutter velocity. In Online Proceedings. 2006 European Wind Energy Conference and Exhibition, Athens (GR), 2006.

[17] Don W. Lobitz. Aeroelastic stability predictions for a MW-sized blade. Wind Energy, 7(3):211-224, 2004.

[18] M.H. Hansen. Aeroelastic instability problems for wind turbines. Wind Energy, 10(6):551-577, 2007.

[19] M.H. Hansen. Aeroelastic stability analysis of wind turbines using an eigenvalue approach. Wind Energy, 7(2):133-143, 2004.

[20] Leonardo Bergami. Aeroservoelastic stability of a 2D airfoil section equipped with a Trailing Edge Flap. Technical Report 1663, Ris National Laboratory, Roskilde (DK), 2008.

[21] Morten Hartvig Hansen, Mac Gaunaa, and Helge Aagaard Madsen. A Beddoes-Leishman type dynamic stall model in state-space and indicial formulations. Technical Report 1354, Ris National Laboratory, Roskilde (DK), 2004.

[22] Thomas A. Zeiler. Results of Theodorsen and Garrick revisited. Journal of Aircraft, 37(5):918-920, 2000.

[23] R.T. Jones. The unsteady lift of a wing of finite aspect ratio. Technical Report 681, National Advisory Committee for Aeronautics (United States Advisory Committee for Aeronautics), 1940.

[24] Peter B. Andersen and Mac Gaunaa. Load reduction using pressure difference on airfoil for control of trailing edge flaps. In EWEC 2009 Proceedings online. 2009 European Wind Energy Conference and Exhibition. Marseille (FR), 2009.

[25] Randall J. Allemang. The modal assurance criterion - Twenty Years of Use and Abuse. Sound and Vibration, 37(8):14-23, 2003.

[26] Mac Gaunaa. Unsteady Aerodynamic Forces on NACA 0015 Airfoil in Harmonic Translatory Motion. PhD thesis, Technical University of Denmark, 2002.

[27] Katsuhiko Ogata. Modern Control Engineering. Prentice-Hall, 1997. 
[28] Peter B. Andersen. Load alleviation on wind turbine blades using variable airfoil geometry (2D and 3D study). Master's thesis, Technical University of Denmark. Department of Mechanical Engineering, 2005. 